\title{
Ecosystem and Biodiversity Monitoring: Best Practice in Europe and Globally (EO4Hab)
}

\section{Stefan Lang and Lena Pernkopf (Eds.)}

This publication is part of the joint dissemination strategy of the EU-funded projects

MS.MONINA (FP7-SPA-2010-1-263479) and BIO_SOS (FP7-SPA-2010-1-263435). It reflects the

views only of the authors, and the Commission cannot be held responsible for any use which may be made of the information contained therein.

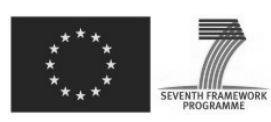

